\title{
Rankings as coordination games: the Dutch Top 2000 pop song ranking
}

\author{
Aloys Prinz ${ }^{1}$
}

Received: 2 July 2015/Accepted: 29 February 2016/Published online: 16 March 2016

(c) The Author(s) 2016. This article is published with open access at Springerlink.com

\begin{abstract}
The Dutch Top 2000 pop song ranking has been broadcast each year since 1999 between Christmas and New Year's Eve. As a nationwide event, it receives cult status in the Netherlands. In this paper, it is argued that rankings are coordination games. To demonstrate this, statistical methods are applied, first to analyze the extent to which competition prevails between pop songs. While conventional charts collect pop song rankings on a commercial basis, the Top 2000 ranking is voted on once a year. Moreover, a very wide range of songs is allocated to 2000 positions. The ranking displays tremendous stability among the top five songs over the years and demonstrates the importance of superstardom. Moreover, a detailed statistical analysis provides evidence that there is hardly any competition among the most liked 100-150 songs. The empirical distribution of these songs follows a Pareto distribution, whereas the songs up to rank 1500 are exponentially distributed. These different distributions may be explained by two different voting strategies, namely voting for the individually most liked songs and voting to maximize the social value of the Top 2000 by taking account of the assumed preferences of other voters. In this manner, superstardom acts as a coordination device in a large-scale coordination game.
\end{abstract}

Keywords Pop charts - Superstars - Coordination games - Pareto distribution · Exponential distribution · Voting strategies

JEL Classification Z110

Aloys Prinz

Aloys.Prinz@wiwi.uni-muenster.de

1 Institute of Public Economics, University of Münster, Wilmergasse 6-8, 48143 Münster, Germany 


\section{Introduction}

Nowadays, not only pop songs and books are ranked, but also countries, universities, scientific journals, and even single scientists. There seems to be a genuine public interest in knowing how other people rate almost everything. In contrast, not so much is known of the meaning of rankings. In this paper, it is argued that rankings are coordination games. The players in this game are people who want to coordinate a social activity that has characteristics of both a private good and a public good. The respective social activity may be a productive process, for instance, scientific work, or a leisure event such as listening to pop music. The private good of the activity may be individual status (in productive processes) or simply personal enjoyment (in leisure events). However, the social activity also has characteristics of a public good, as it requires many coordinated activities to be productive or to be a fully enjoyable event. In leisure events, the good itself-the "event"-is collectively provided by the participants themselves (see DeSerpa and Faith 1996, who dubbed such goods "mob goods").

Rankings as such, and especially rankings as coordination games, raise two related questions. Firstly, how stable and contestable are rankings? Superstars, for instance, are those people or institutions who are at the top of rankings, in some cases for a long time. Although there is an economic theory of superstardom (Rosen 1981; Adler 1985; MacDonald 1988), it neither is known whether the superstar position is stable nor whether it is contestable. The second question is how these rankings and their contestability can be explained.

To answer these questions, in this paper, the Top 2000 charts of the Dutch public NPO Radio 2 (www.radio2.nl/top2000) are analyzed. This chart started in 1999 and has become very popular until today. The chart is based on a voting by radio listeners, i.e., it is not a ranking of songs according to commercial success. Rather, it looks for the 2000 "most meaningful songs to our audience," as pointed out by the broadcaster (NPO Radio 2 2015a). The songs are ranked according to votes and are broadcast (starting with position 2000) from Christmas Day to midnight on New Year's Eve. The Top 2000 is a big social event nationwide in the Netherlands.

The data of all rankings from 1999 to 2014 are available on the Web site of NPO Radio 2 (2014). The song titles, the artists, and the year of song production are all indicated. Almost the same dataset (i.e., the data without 2014) was analyzed recently by Sterken (2014). Sterken's main objective was to analyze the impact of the year of song production on its ranking. The "loss of collective memory" effect was juxtaposed with the "nostalgia" effect of pop songs. Additionally, Sterken (2014) explained empirically the life span of songs by means of the respective artists' superstar status (The Beatles, The Rolling Stones, and several others are present in the ranking with a large number of songs), the songs' lengths and language, as well as their debut rankings.

The dataset of the Top 2000 songs and their rankings over time are an unusual source for studying the dynamics of popularity and success in popular music. Charts and bestseller lists are normally very short (about ten to 100 ranks). Consequently, they represent only a small fragment of the respective market, so that the 
distribution of popularity and success can only be measured for the very top level. In addition, charts measure mainly commercial success. In contrast to music charts and bestseller lists, the Top 2000 event takes place annually, and the song rankings are voted on by a large part of the Dutch population. Hence, the rankings represent a much greater share of the popular music market. Moreover, song success in the Top 2000 charts is not commercially defined and motivated. Hence, this data basis allows for a much broader analysis, both statistically and economically.

In this paper, the distribution of song success over all rankings, as well as the underlying stochastic process, is studied. The first objective is to determine the contestability of the song ranking. Although this issue is related to the topics studied in Sterken (2014), the objective is nevertheless quite different. The second objective of the paper is to provide a rationale for the distribution of the popularity of songs in the Top 2000 charts. Since the database of these charts is large, the distribution of song success can be analyzed thoroughly. Moreover, the statistical results constitute a solid basis for economic interpretation. The result is that the Top 2000 ranking is seen here as a coordination game of the Dutch population.

The remainder of the paper is structured as follows. In the second section, the relevant literature on the distribution of success in the industry of popular music is reviewed. Section 3 contains the descriptive statistics of the bilateral ranking correlations for the years 1999-2014, as well as an analysis of the empirical distribution of song success. In Sect. 4, the empirical results are discussed and interpreted. Section 5 concludes.

\section{Literature review}

Besides the paper of Sterken (2014), there is an economics literature on success and popularity in popular music. The industry is an integral part of modern culture. According to Statista (2015), the global revenue of the music industry in 2012 was US-\$ 47.4 billion, of which US-\$ 15.2 billion were generated in the USA [for the economics of the music industry, see Burke (2011); an economic analysis of the rock and roll industry is provided by Connolly and Krueger (2006); they also discuss ranking methods].

The cultural aspect of songs is emphasized by the unpredictability of success even by experts, the partial influence of "quality" on success, as well as the inequality of success. In a study of Salganik et al. (2006), for instance, "quality" was measured by the ranking of songs by participants, but without any information about the ranking decision of other participants. Haan et al. (2005) compare the "quality" judgment of experts with the judgment of the public opinion in the national finals of the European Song Contest. Ginsburgh and Noury (2008) measure "quality" in the same contest by excluding votes of the same "voting bloc" via the result of the contest. Ginsburgh (2003) compares "types of evidence" for "quality" measured with prizes awarded by experts, economic success, and long-term reputation of the artwork (which is assumed as a key variable for aesthetic quality). The success variable applied in this paper is similar to Ginsburgh's long-term 
reputation since success will be defined via the number and ranks of appearances in the Top 2000 rankings over more than a decade.

A larger number of papers study the Eurovision Song Contest that started in 1955 and are still running each year. However, the main topic of these papers is whether there exist voting blocs (see, e.g., Yair and Maman 1996; Fenn et al. 2006; Gatherer 2006; Spierdijk and Vellekoop 2009), friends, and foes (García and Tanase 2013, who also measure cultural affinity among contest countries and vote polarization), as well as if political considerations affect voting (among others, Ginsburgh and Noury 2008). According to these studies, although voting blocs due to cultural affinity and geographical proximity seem to exist (Ginsburgh and Noury 2008; Spierdijk and Vellekoop 2009), evidence for political voting is rather mixed, but it became probably more likely in the recent years of European crises (García and Tanase 2013).

Apparently, songs can be divided into two categories only, "the best" and "the rest" (Salganik et al. 2006). The latter means that the distribution of success is highly concentrated on a small number of "hits." Therefore, pop music can be regarded as an excellent field for research on superstardom (for economic analyses of superstars, see Rosen 1981, 1983; Adler 1985, 2006; MacDonald 1988; Schulze 2011).

Superstardom in popular music is a topic in Hamlen $(1991 ; 1994)$. The superstar hypothesis of Marshall (1947) and Rosen (1981)—small differences in talent ("quality") make a big difference in success-could not be confirmed by Hamlen (1991). About 100 singers are analyzed, with the total value of record sales as the measure of success. Although "voice quality" played a role in success, its estimated coefficient was too small to confirm a superstar effect. Differentiating between the market for "singles" (as an "entry-level quality"; Hamlen 1994, p. 395) and "albums" did not change this result (Hamlen 1994). Chung and Cox (1994) took a statistical approach to modeling the superstar phenomenon. They showed that a stochastic model, applying the Yule distribution (Yule 1924; Simon 1955), generates a distribution of success with a small number of winners. This model was supported by music industry data for gold records of 1377 performers between 1958 and 1989 (Chung and Cox 1994, p. 773). The conclusion of Chung and Cox was that superstardom could indeed exist without differences in talent.

Since the definition of "success" is crucial for the measurement of stardom, Giles (2006) employs the number of weeks a number-one hit retains (not necessarily consecutively) this spot as a measure of success. Data for 1955-2003 are used, with more than 900 observations. As a second success measure, Giles applies the number of number-one hits by artists. Only 245 artists (of a total number of 429 artists with at least 13 recordings in the charts) gained number ones during the respective time period. The result, however, was that the Yule distribution does not fit the data well if number-one hits (in both versions) are taken as measures of success. In an earlier study, Cook (1989) did not find statistically significant relationships between the observed distribution of the Billboard Top 40 single chart artists and the Lotka (1926) as well as the Bradford (1934) distribution.

Also, Fox and Kochanowski (2004) did not find a stochastic superstar effect for single records that gained gold or even platinum (single) awards in the USA 
between 1958 and 2001, when applying the Lotka (1926) and Yule (1924) distribution to test for superstardom. The result changes if a so-called generalized Lotka distribution is applied (Fox and Kochanowski 2004, pp. 516 ff.). The application of "Lotka's law" to analyzing the distribution of gold records, studied by Cox et al. 1995, reached results that are very similar to those of Fox and Kochanowski, as the "generalized Lotka law" provided a good fit. However, as pointed out by Fox and Kochanowski, a major problem with stochastic approaches is that they do not say anything about the underlying processes that generate the statistical distribution (Fox and Kochanowski 2004, p. 518).

Popular music chart success in the UK (British album charts) for 1980-1993 was studied by Strobl and Tucker (2000). The substantial skewness of all measures of album success in the charts was, among others, also driven by the initial popularity of the respective album. However, the Yule distribution was rejected as the underlying stochastic process for success (Strobl and Tucker 2000, p. 124). Although no dynamic process was studied, an analysis of the demand for top popular music singles and albums in Japan provided empirical evidence of a highly skewed cumulative share of singles and albums in the Top 100 charts in 2007 and 2008 (Asai 2011, p. 124).

Crain and Tollison (2002) present a model-based estimation of the concentration of top artists in the popular music industry. The crucial notion is that stardom may be a device for economizing on time that customers devote to seeking people with similar music preferences (Crain and Tollison 2002, p. 4). Data from Billboard Magazine on the top four and top five artists who were ranked number one are employed. An artist concentration index was defined as the proportion of weeks of the year that those artists were ranked number one. Customers' average weekly earnings are the key variable for determining the concentration index, because they reflect customers' time opportunity costs. As a result of the estimations, higher earnings are Granger-causal for higher concentration values. Hence, the authors conclude that time economizing for consumers is the driving force of stardom.

Taking account of incomplete consumer information, Hendricks and Sorensen (2009) explain the skewed demand for popular music via a learning-based model. Due to a lack of information about artists, a new album release of an artist that becomes a hit causes a so-called backward spillover as sales of the artist's old albums increase permanently after the new album's success. As a consequence, the demand for popular music is far more skewed with incomplete than with complete information.

The "lifetimes" of songs was statistically analyzed by Bradlow and Fader (2001). To describe the lifetime of the Hot 100 Billboard songs, the authors assumed a generalized gamma lifetime curve. An interesting result was that a popular song falls quickly once it leaves the Top 40. Moreover, a 20-week life span separated different exit patterns for songs, and the turnover of songs was not smooth (Bradlow and Fader 2001, p. 378).

The stochastic dynamics of the music album lifecycle was studied by Bhattacharjee et al. (2007). Due to the advent of file sharing and other such processes, album time in the charts has shortened. However, albums that survive the first few weeks fare much better afterward, staying longer in the charts. 
To sum up, a number of studies emphasize the stochastic nature of success in the popular music industry. However, the measure of success used is crucial, as the results differ for different success measures. Moreover, although a number of different candidate distributions were checked to describe the distribution of success statistically, the results are ambiguous. Nevertheless, even if the stochastic nature of pop song success is well established, the underlying economic process does not seem to be well understood.

\section{Empirical analysis}

\subsection{Descriptive data analysis}

The Top 2000 charts have been broadcast by the Dutch public NPO Radio 2 each year since 1999, between Christmas and New Year's Eve (December 25-31). The charts contain the ranking of 2000 pop songs, collected via a not entirely publicly available voting mechanism (see Sterken 2014, and the sources given therein). The songs are predominantly in English $(83.5 \%)$, but some have also been in Dutch $(9.9 \%)$, French $(1.6 \%)$, Italian $(0.9 \%)$, German $(0.7 \%)$, Spanish $(0.6 \%)$, and Portuguese $(0.1 \%)$ over the years (Sterken 2014, p. 6; Table 1; percentages rounded here; note that the percentages are calculated without 2014).

Table 1 Number of songs by artists and bands with the largest number of songs in the Top 2000 (2014)

Source: http://www.radio2.nl/ nieuws/id-6066-top-2000-factsfigures.html (Accessed November 05,2015 )

\begin{tabular}{ll}
\hline Artist & 2014 \\
\hline The Beatles & 41 \\
The Rolling Stones & 30 \\
Queen & 24 \\
Michael Jackson & 22 \\
ABBA & 21 \\
U2 & 21 \\
Bruce Springsteen & 20 \\
Coldplay & 19 \\
Fleetwood Mac & 18 \\
Bløf & 16 \\
De Dijk & 15 \\
David Bowie & 15 \\
Elvis Presley & 14 \\
Anouk & 14 \\
Dire Straits & 13 \\
Bee Gees & 12 \\
Creedence Clearwater Revival & 12 \\
Golden Earring & 12 \\
Ilse DeLange & 12 \\
Marco Borsato & 12 \\
\hline
\end{tabular}


The number of song voters grew from 150,000 in 1999 to 3.3 million in 2013 (Sterken 2014, p. 4) and 3.8 million in 2014 (http://www.radio2.nl/ebu/?page=t2kimpact, Accessed November 05, 2015). That is, based on the Netherlands population aged between 20 and 65 in 2009 (Statistics Netherlands 2015), this yields participation rates of $1.5,32.7$, and $37.7 \%$, respectively. Hence, a substantial part of the eligible Dutch population participated in the voting. However, according to a statistical analysis for 2010 (Goris 2010), $62 \%$ of the voters were men so that the Top 2000 voting is male-dominated.

Figure 1 shows the age distribution of voters in 2010. It is bimodal, with the first peak for the age group of 16-20 years and the second between 46 and 50 years (Goris 2010). About 59.4\% of voters are aged between 41 and 60 .

Between 1999 and 2008, voters could choose ten songs, from 2009 onwards they could choose 15 (Sterken 2014, p. 5). The choice of songs was entirely open in 1999 and 2000, but restricted afterward. The restricted choice consists of the titles of the 1999 and 2000 polls, as well as of the record lists of NPO Radio 2 for the respective year (Sterken 2014, p. 5). However, Sterken (2014, p. 5) claims that this did not change the chart's composition, according to NPO Radio 2.

In all 16 years of the Top 2000, a total of 3906 songs competed for the 2000 available ranks; i.e., the relationship of songs to ranks is 1.953 . New songs are given a chance to make it to the Top 2000 charts if they replace another song that is already listed. Figure 2 shows the distribution of publication years of songs in the pool of the Top 2000 songs.

The range of publication years starts in 1924, with George Gershwin's "Rhapsody in Blue," and ends for this analysis in 2014; the median publication year is 1979, and the mean year is 1982 with a standard deviation of 14.62 years. Figure 3 demonstrates that the publishing years of songs are not distributed uniformly over the years; songs before the year 1960 and those published between 1970 and about 1990 are overrepresented. Taking account of the age structure of voters in Fig. 1, the age of songs corresponds to some extent with voter age (see also Sterken 2014).

The frequencies of average song ranks ("avgsucc") in the Top 2000 charts are presented in Fig. 4.

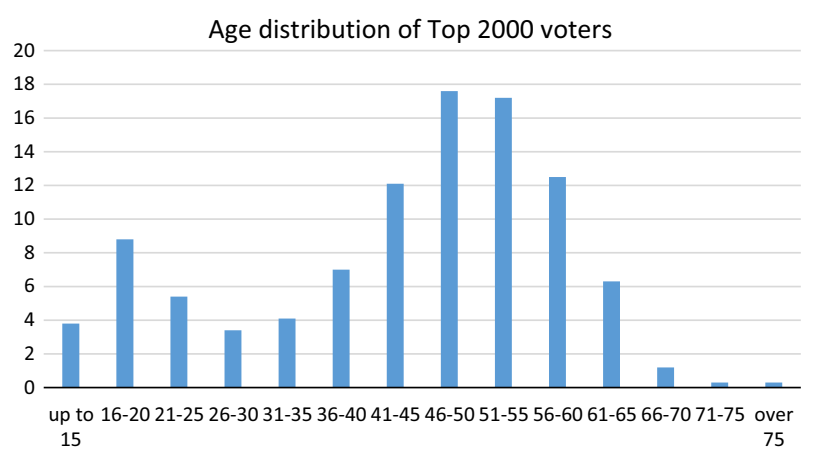

Fig. 1 Age distribution Top 2000 voters (women and men). Source: Goris (2010), p. 39 


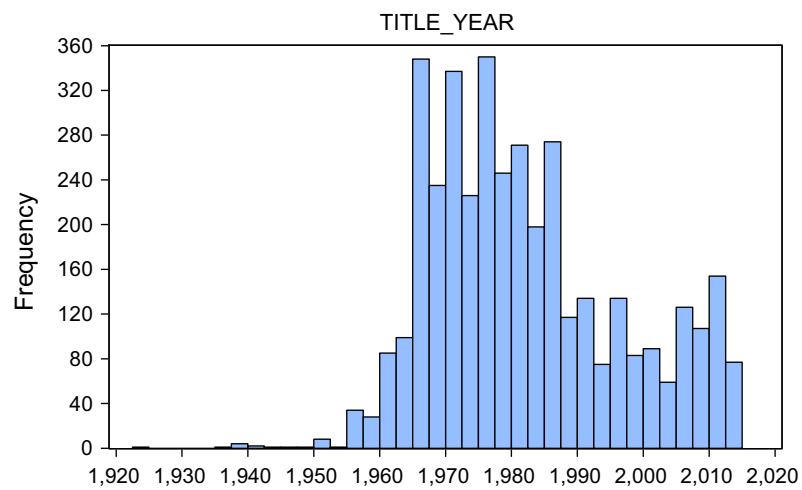

Fig. 2 Distribution of Top 2000 songs according to publication year. Source: Own calculations with data from NPO Radio 2 (2014)

Fig. 3 Uniform versus empirical distribution quantiles of song publication years. Source: Own calculations with data from NPO Radio 2 (2014)

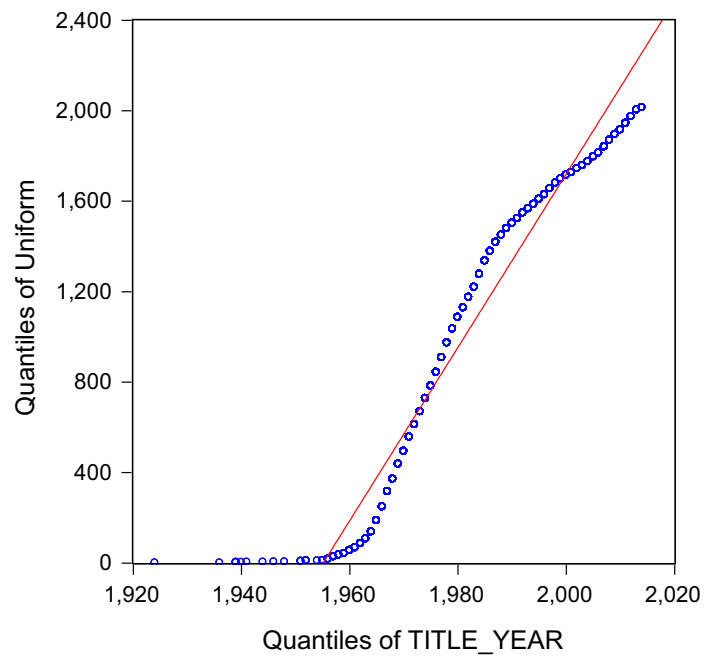

The median song rank is 1373 and the mean rank 1225, with a standard deviation of 539.28 ranks. Figure 5 demonstrates that the distribution of ranks is not uniform and that there are thick tails at the highest and lowest ranks.

A general tendency, already mentioned in the reviewed literature, is the concentration of songs by artists in pop charts. This is also true for the Top 2000 (Table 1). In the Top 2000 of 2014, The Beatles, The Rolling Stones, and Queen are those bands with the highest number of ranked songs.

Moreover, since 2004, the top 5 songs did not change; only their ranking varied. These top 5 songs are (in the order of the Top 2000 ranking in 2014) as follows: "Hotel California," The Eagles (1977); "Bohemian Rhapsody," Queen (1975); "Stairway to Heaven," Led Zeppelin (1971); “Child in Time,” Deep Purple (1972); 


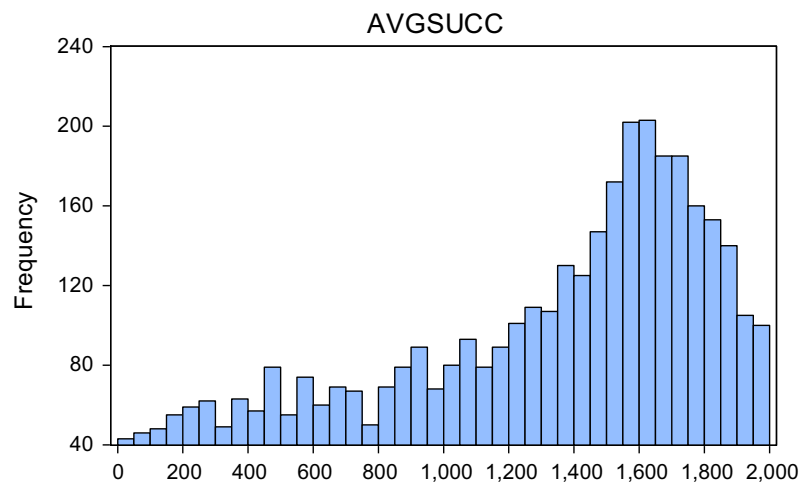

Fig. 4 Frequencies of average ranks in the Top 2000. Source: Own calculations with data from NPO Radio 2 (2014)

Fig. 5 Uniform versus empirical distribution quantiles of song ranks. Source: Own calculations with data from NPO Radio 2 (2014)

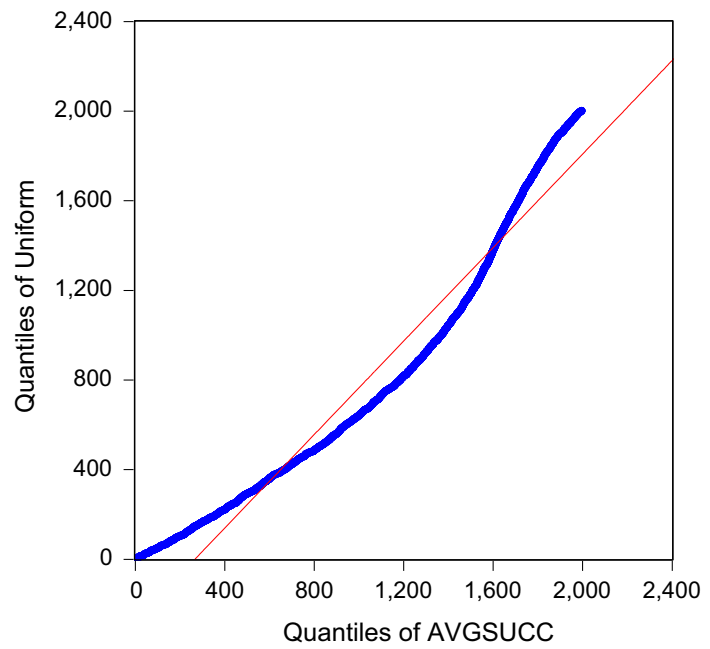

and "Avond," Boudewijn de Groot (1997). Except for the Dutch song "Avond," all songs were published in the first half of the 1970s.

For a further descriptive analysis, Spearman's rank-order correlation coefficients of all years' chart rankings are shown in Table 2.

Only four values of Spearman's rank-order correlation coefficient are statistically insignificant at the $1 \%$ level (rank 2009, rank 2000; rank 2010, rank 2000; rank 2013, rank 2001; and rank 2014, rank 2002). A general tendency is that the correlation coefficient decreases as the year differences increase. Even some correlation coefficients change sign and become negative. Obviously, there is a connection among the rankings over years, but it declines over the years. 
Table 2 Spearman's rank-order correlation coefficients of all songs for all years

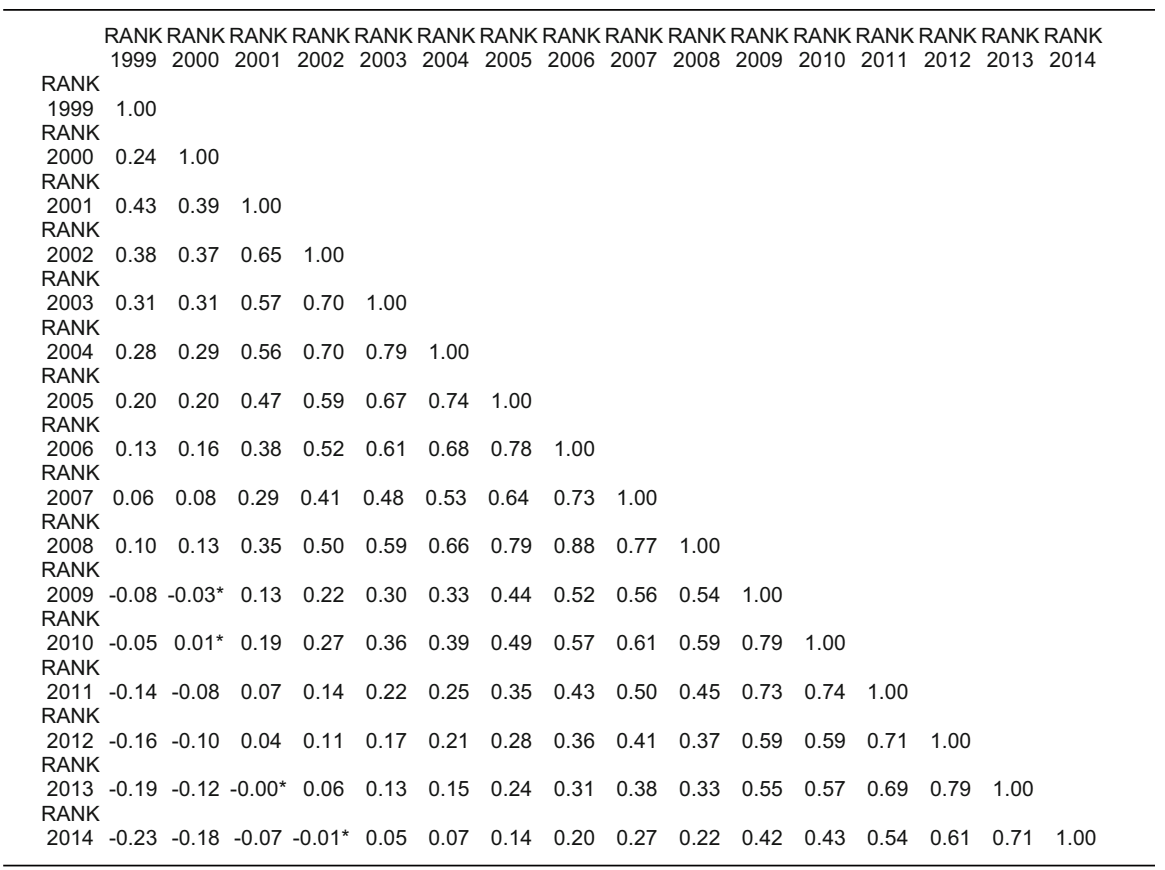

Values with an asterisk * are not statistically significant at the $1 \%$ level

Source: Own calculations with data from NPO Radio 2 (2014)

\subsection{Distribution of song success}

In order to analyze the distribution of song success, its measurement must be defined. As shown by Simkin and Roychowdhury (2013) with empirical data, the logarithm of "fame" seems to be an adequate measure of "achievement" (see also the "Celebrity Index" in Lamport 2006). Therefore, the following definition is employed for the analysis in this paper:

$$
\begin{aligned}
X_{i} & =\left[\log \left(\left(\# \operatorname{Rank}_{i j}>0\right) \cdot\left(\sum_{j=1}^{\# \operatorname{Rank}_{i j}>0} \operatorname{Rank}_{i j}\right)^{-1}\right)\right]+C, \quad j=\text { year } \\
j & =1999, \ldots, 2014 .
\end{aligned}
$$

The success of song $i, X_{i}$, is the natural logarithm of the mean rank the song occupied in all the years it had a rank greater than zero, i.e., the number of years it was ranked in the Top 2000 charts. Moreover, a constant $C=7.7$ is added to obtain success values that start only at about zero (this means that the success of the song ranked 2000 is about zero). The distribution of the success values, calculated via Eq. (1), is shown in Fig. 6.

The question is as follows: Which distribution function does the distribution of song success, as depicted in Fig. 6, fit best? As a first step in finding this out, 


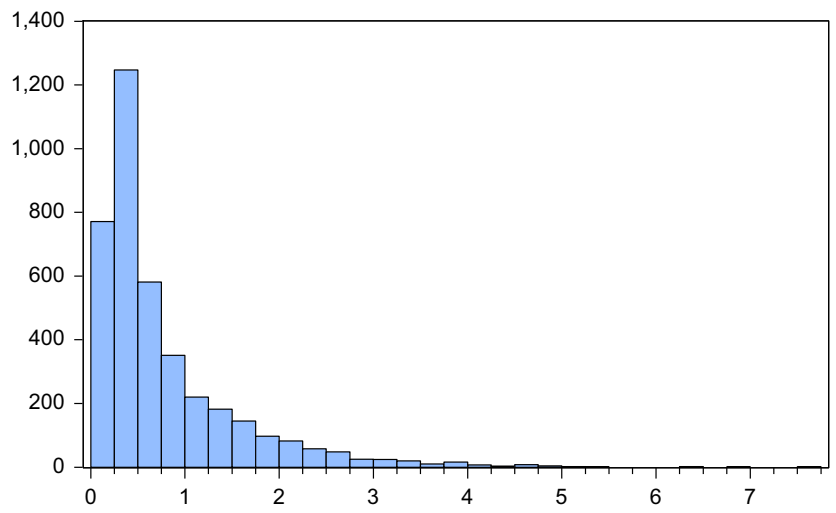

\begin{tabular}{|ll|}
\hline \multicolumn{2}{|l|}{ Series: SUCCESS } \\
Sample 1 3906 \\
Observations & 3906 \\
& \\
Mean & 0.779680 \\
Median & 0.475083 \\
Maximum & 7.528150 \\
Minimum & 0.099598 \\
Std. Dev. & 0.785728 \\
Skewness & 2.429862 \\
Kurtosis & 11.23484 \\
& \\
Jarque-Bera & 14880.16 \\
Probability & 0.000000 \\
\hline
\end{tabular}

Fig. 6 Histogram of song success values. Source: Own calculation with data from NPO Radio 2 (2014).

$Q-Q$ plots are generated. ${ }^{1}$ In these plots, the success variable's quantiles of the empirical distribution are juxtaposed against the respective quantiles of the theoretical distribution that is tested. EViews estimates the parameters of the theoretical distribution via maximum likelihood estimations. In Fig. 7, the $Q-Q$ plots for the theoretical exponential distribution are presented (for a statistical analysis of popularity, see Pan and Sinha 2010). ${ }^{2}$

The figure indicates that the exponential distribution fits the empirical distribution of the success variable well up to a point. For major success, the fit of the exponential distribution is not good. The fit of the other distributions mentioned in footnote 2 is also not good. Nonparametric tests of the respective distributions against the empirical distribution confirm that the respective null hypotheses (i.e., the empirical distribution is an exponential, Pareto, Weibull, gamma, or lognormal distribution) are altogether rejected.

However, the distribution histogram of the success variable seemingly hints at a sort of extreme distribution. According to the econophysics concept of "preferential attachment" (see, e.g., Newman 2010, chapters 14.1-14.4, pp. 487 ff.), the data might follow a power law distribution. To test this, the estimation method of Gabaix and Ibragimov (2011) is applied. The equation of estimation is then given by:

$$
\log (i-0.5)=a+b \cdot X_{i},
$$

with $i$ as rank and $a, b$ as estimation coefficients of the Pareto (power law) distribution. ${ }^{3}$ The results of the OLS estimation with "White heteroskedasticity-consistent standard errors and covariance" are shown in Table $3 .^{4}$

\footnotetext{
1 The plots are produced with the econometric software package EViews 8.

2 Q-Q plots of the Pareto, Weibull, gamma, and lognormal distribution (not presented) were also tested, but did not produce better results.

${ }^{3}$ Note that the standard error of the estimated power law coefficient $b$ is asymptotically $(2 / N)^{1 / 2} b(\mathrm{Gabaix}$ and Ibragimov 2011).

${ }^{4}$ It is worth emphasizing that the estimation of the power law coefficient is a descriptive statistic. Therefore, the adjusted R-squared value must be interpreted very cautiously. The reason is that the
} 
Fig. 7 Q-Q plot for the exponential distribution: $f(x \mid a, \mathrm{mu})=\frac{1}{\mathrm{mu}} e^{-\left(\frac{x-a}{\mathrm{mu}}\right)}$. Source: Own calculation with data from NPO Radio 2 (2014)

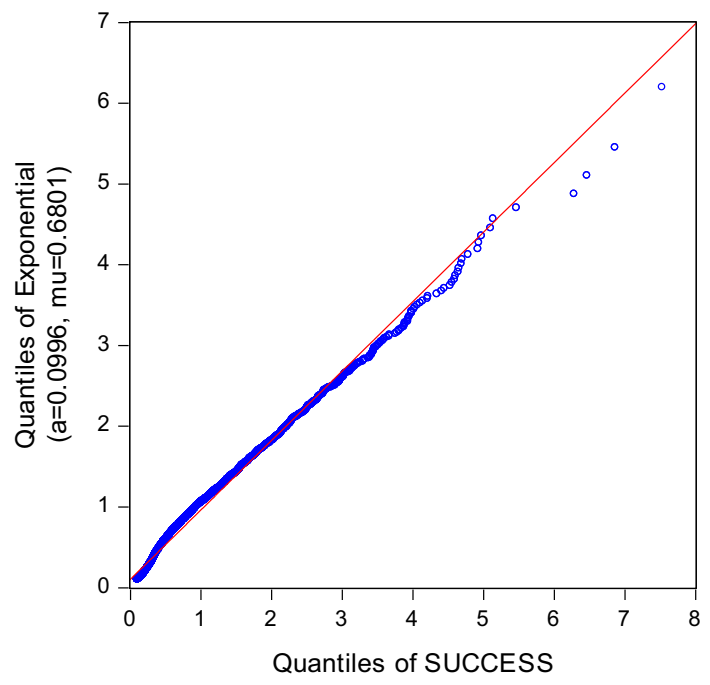

Although the estimation seems to fit the data well, a look at the residuals reveals that there is a systematic deviation of the fitted from the actual data. Moreover, tests of the stability of the estimated coefficients (CUSUM test) in Fig. 8 show that they are not sufficiently stable (at the $5 \%$ level).

To check this more thoroughly, the dataset is split into increasing rank groups. Starting with the ranks up to rank 50, the next group encompasses those up to rank 100 , and so on. In each case, the null hypothesis that the data follow a Pareto distribution (an exponential distribution) is tested. The detailed results are shown in Table 4. Up to about rank 100, the hypothesis of a Pareto (power law) distribution is not rejected (Cramer-Mises test). The most promising alternative hypothesis, that the data follow an exponential distribution, is also tested, and clearly, this distribution fits the data well up to rank 1500; the hypothesis is not rejected (Cramer-Mises test). However, the best fit of the exponential distribution is between the ranks of 100 and 1500 .

The conclusion of these tests is as follows:

1. For the first 50 ranks, the Pareto distribution seems to be the appropriate distribution function.

2. For the ranks between 50 and about 1500 , the exponential distribution gives the best fit.

These results lead to the question of whether it is possible to find a combination of the Pareto (power law) and exponential distributions that may fit the data even

Footnote 4 continued

measure of success is serially correlated; however, since success is defined via the autocorrelation of positions in subsequent rankings, this correlation cannot be eliminated for the estimation of the form of the success distribution function. 
Table 3 Power law coefficients, all data

\begin{tabular}{lclr}
\hline Variable & Coefficient & Standard error & Prob. \\
\hline$A$ & $8.2564 * * *$ & 0.0038 & 0.0000 \\
$B$ & $-1.2647^{* * *}$ & 0.0049 & 0.0000 \\
Adjusted $R^{2}$ & 0.9888 & & \\
\hline
\end{tabular}

$* * *, * *, *$ Statistically significant at the 1,5 , and $10 \%$ level, respectively

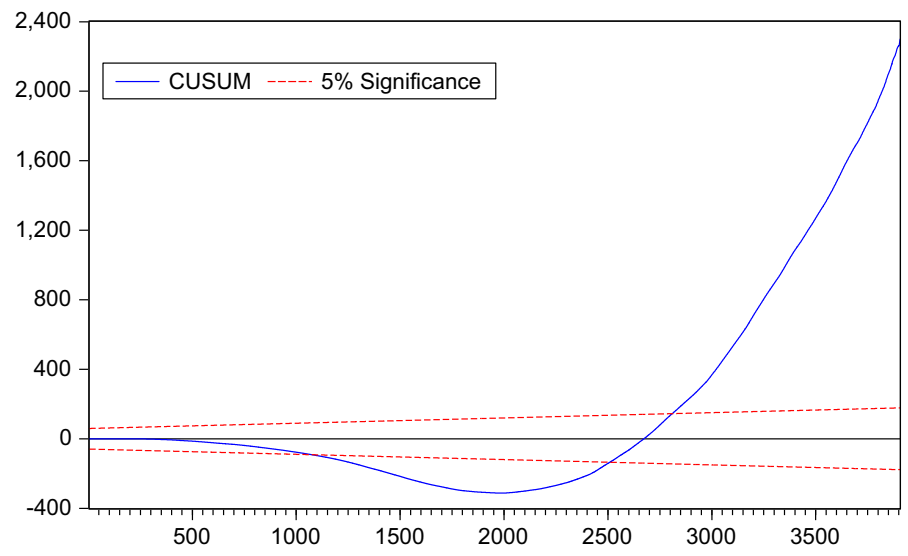

Fig. 8 CUSUM stability test for the power law estimation

better. Meerschaert et al. (2012) proposed the "tempered power law distributions" for so-called "semi-heavy tailed distributions" (p. 1839). The distribution, with $x$ as song success, is defined as (Meerschaert et al. 2012, p. 1841):

$$
f(x)=\gamma \cdot x^{\alpha} \cdot e^{-\frac{\beta}{x}}
$$

which can be written in logarithmic form as follows:

$$
\log f(x)=\log \gamma+\alpha \cdot \log x-\frac{\beta}{x} .
$$

The OLS estimation equation reads:

$$
\log (\text { Rank })=c(1)+c(2) \cdot \log x-c(3) \cdot x^{-1} .
$$

The coefficients of estimation are as follows: $c(1)=7.017, c(2)=-1.8138$, and $c(3)=0.3433$; all coefficients are statistically significant at the $1 \%$ level; the adjusted R-squared value is 0.9505 . The residuals of the estimation are depicted in Fig. 9.

The CUSUM stability test of the estimated coefficients, depicted in Fig. 10, shows that they are stable up to about rank 3000 . The adjusted $R$-squared value of 
Table 4 Test of Pareto and exponential distribution functions

\begin{tabular}{lllll}
\hline Rank & $\begin{array}{l}\text { Pareto } \\
\text { distribution }\end{array}$ & $\begin{array}{l}\text { Cramer-Mises } \\
\text { probability }\end{array}$ & $\begin{array}{l}\text { Exponential } \\
\text { distribution }\end{array}$ & $\begin{array}{l}\text { Cramer-Mises } \\
\text { probability }\end{array}$ \\
\hline$<51$ & $K=3.5871$ & 0.2078 & $\begin{array}{l}A=4.9196 \\
\mathrm{MU}=0.8461\end{array}$ & 0.0878 \\
& $A=5.0851$ & & $A=3.5871$ & 0.5431 \\
$<101$ & $K=2.9865$ & 0.0784 & $\mathrm{MU}=0.8459$ & \\
& $A=4.2843$ & & $A=2.9865$ & 0.8346 \\
$<151$ & $K=2.6390$ & 0.0443 & $\mathrm{MU}=0.8613$ & \\
& $A=3.9062$ & & $A=1.5650$ & 0.9544 \\
$<501$ & $K=1.5650$ & 0.0000 & $\mathrm{MU}=0.8794$ & \\
& $A=2.5261$ & & $\mathrm{~A}=0.9585$ & 0.5675 \\
$<1001$ & $K=0.9585$ & 0.0000 & $\mathrm{MU}=0.8840$ & \\
& $\mathrm{~A}=1.7635$ & & $A=0.6462$ & 0.1611 \\
$<1501$ & & & $\mathrm{MU}=0.8473$ & \\
& & & $A=0.4635$ & 0.0000 \\
$<2001$ & & & $\mathrm{MU}=0.7936$ & \\
& & & &
\end{tabular}

Pareto distribution: $K$ and $A$ are parameter of the function: $f(x \mid A, K)=\left(A \cdot K^{A}\right) / x^{A+1}$. Exponential distribution: $A$ : parameter of this distribution, MU: mean of the function $f(x \mid A, \mathrm{MU})=\frac{1}{\mathrm{MU}} e^{-\left(\frac{x-A}{\mathrm{MU}}\right)}$. These distributions are implemented in EViews 8.

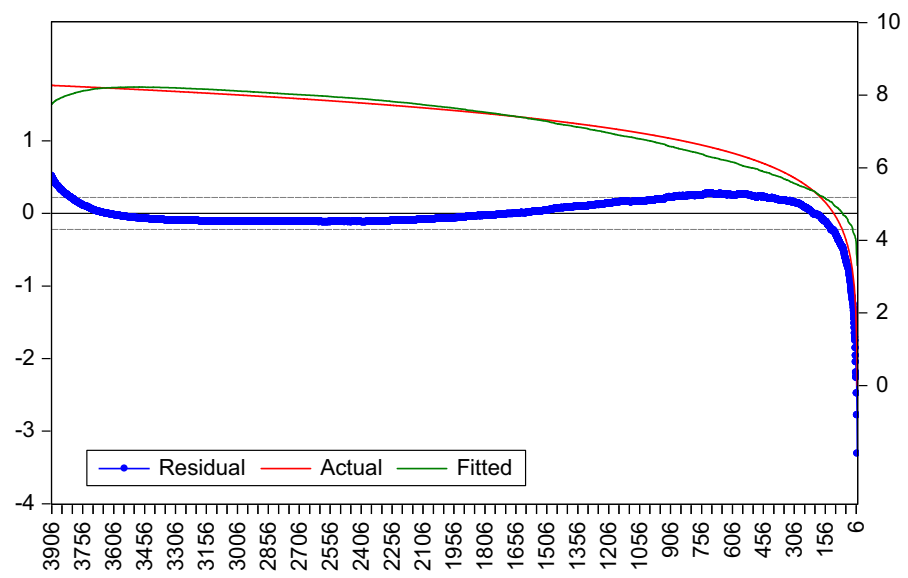

Fig. 9 Residuals of the tempered power law estimation

the power law estimation is also slightly higher than that of the tempered power law estimation, and the CUSUM test implies a higher stability for the latter.

Hence, the distribution of song success in the Dutch Top 2000 charts seems to follow a power law tail, combined with an exponential tempering.

The results presented here are similar to those of Chung and Cox (1994); they also found a stochastic superstar effect in their analysis, but for a different success 


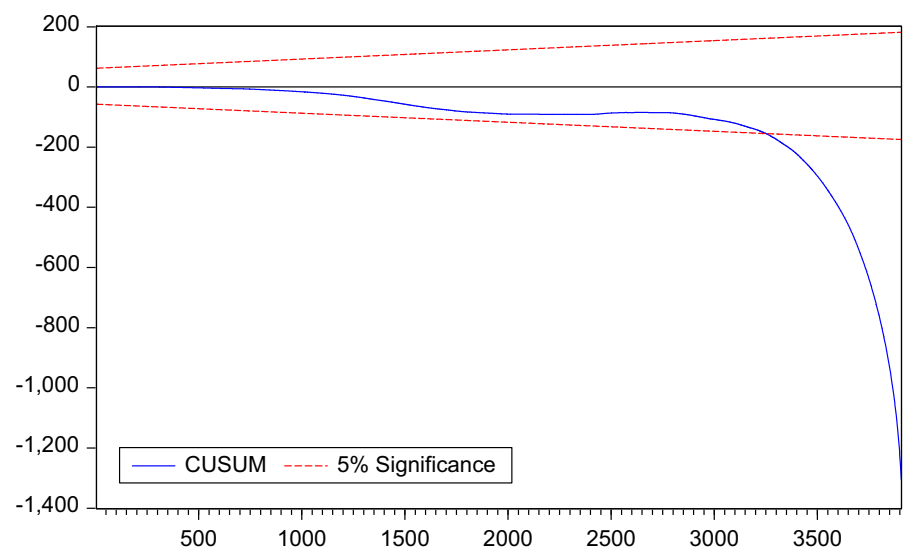

Fig. 10 CUSUM stability test for the tempered power law estimation

measure. The paper of Fox and Kochanowski (2004) - that could not find unambiguous evidence for stochastic superstardom-is not directly comparable with this paper since the authors applied the number of gold and platinum records as a success measure. Since the measure applied in this paper resembles more that of Giles (2006), the result of this paper is comparable to the latter. Giles could not find superstardom when analyzing the life lengths of number-one hits in the Billboard Hot 100 chart. In contrast, in the Top 2000 charts, a superstar effect is present, with the top five songs at least.

\section{Interpretation of the empirical results}

According to Prinz et al. (2015), let $Y_{i}=\frac{1}{X_{i}}$ be the success measure of a pop song in the Top 2000 charts for song $i, i=1, \ldots N$, such that $i$ is also the rank of the song: ${ }^{5}$

$$
Y_{1}>Y_{2} \cdots>Y_{N}
$$

Song success can be modeled as a dynamic stochastic process in which competition between songs for listeners' votes plays a crucial role. If the songs were equally contestable, there would be a strong so-called reversion to the mean effect that sets a limit to song success. As a consequence, a large number of songs should in fact be represented in the rankings over time, with no extremely long presence of songs at the top positions of the ranking.

A general mean-reverting stochastic process that represents the dynamics of song success can be written formally as (Prinz et al. 2015):

\footnotetext{
5 This kind of formalization and the following stochastic analysis closely follows Prinz et al. (2015), Sect. 4.
} 


$$
d Y_{i}(t)=\theta \cdot\left(\mu-\lambda \cdot Y_{i}(t)\right) \cdot\left(\frac{Y_{i}(t)}{\mu}\right) d t+\sigma \cdot Y_{i}(t) \cdot d W(t),
$$

with $d Y_{i}(t)$ as the change in $Y_{i}$ over time $t, \mu(>0)$ as the mean value of the success variable, $Y_{i}, \theta(>0)$ as the speed parameter of reversion to the mean, and $0 \leq \lambda \leq 1$ as the value with which the success variable, $Y_{i}$, itself contributes to the meanreverting process. Furthermore, $\sigma(>0)$ is the variance of $Y_{i}$, and $d W(t)$ is a standard Wiener process with a mean of zero and standard deviation $(d t)^{1 / 2}$. Note that the dynamic stochastic process described by (10) implies that for $\lambda>0$, the change in success is proportional to the level of success and, hence, a multiplicative stochastic process. ${ }^{6}$

In this dynamic process, the mean success value, $\mu$, represents the long-term success equilibrium of a contestable song market. The value of $\theta$ indicates the speed of convergence to the mean song success value; hence, it can be interpreted as the contestability of the song market. Moreover, the value of $\lambda$ determines to what extent large success values, in relation to mean success, have (or have no) influence on the reversion to mean success. The smaller the $\lambda$, the lower the impact of very successful songs on the reversion to the mean, and the higher the invasion of new songs into the charts. Consequently, a value of $\lambda$ near to unity implies that there is almost no innovation in the charts.

In applying the dynamics defined by Eq. (7) to the Top 2000 charts, it is very helpful that the resulting distributions of success values for different parameter values of $\lambda$ and $\theta$ are known (for details, see Prinz et al. 2015). Table 5 shows the constellation between the stochastic process and the distribution of the success variable it generates.

As indicated in Table 5, the value of $\lambda$ is crucial for defining the specific stochastic process. Since it is well known from the mathematical literature that certain stochastic processes imply specific distributions of the variable in question (here, the success of songs in the Top 2000 charts), the Top 2000 rankings data can be employed to test which of the distributions provides the best data fit. Knowing the most likely empirical distribution, the generating stochastic process may be discovered. From the stochastic process, it can then be inferred whether the song ranking is contestable (for this procedure and its application to the ranking of art galleries, see Prinz et al. 2015).

At first glance, the results of the estimations in the previous section are inconclusive. If a combination of two different distributions provides the best data fit, the question is whether there is a single stochastic process that generates the empirical data. Interestingly, the distribution of income and wealth seems to be best characterized empirically by a combination of the exponential distribution for a very large majority of the population and the Pareto (power law) distribution for very high levels of income and wealth (Drăgulescu and Yakovenko 2001; for an overview, see Yakovenko and Rosser 2009). The stochastic processes that govern

\footnotetext{
${ }^{6}$ For the effect of this characteristic of the stochastic process for the formation of opinions and collective behavior, see Bahr and Passerini (1998).
} 
Table 5 Stochastic processes and their distribution functions

\begin{tabular}{ll}
\hline Stochastic process & Distribution of $Y_{i}$ \\
\hline Geometric Brownian motion $(\lambda=0, \mu=\theta)$ & Lognormal distribution (with mean $=\theta)$ \\
$\begin{array}{l}\text { Mean-reverting geometric Ornstein-Uhlenbeck process } \\
(0<\lambda \leq 1)\end{array}$ & For $2 \theta>\sigma^{2}:$ Gamma distribution ${ }^{\mathrm{a}}$ \\
Inhomogeneous geometric Brownian motion $(\lambda=1)$ & $\begin{array}{c}\text { Pareto distribution (also called power law } \\
\text { distribution) }\end{array}$ \\
\hline
\end{tabular}

Source: Compiled from Prinz et al. (2015)

a Note that the exponential distribution (also applied to the data) is a special case of the gamma distribution

the distribution of income and wealth, as well as the success of pop songs in the Top 2000 contest, seem to be very similar.

The reason for this similarity might be purely accidental; however, the distribution of income and wealth, as well as the success of popular music (and other forms of artwork), is the result of a combination of talent, performance, and luck. To become very rich, as well as to get to the top of the charts, involves many successful steps in-between, each of them very unstable (see Prinz 2015). Such a process implies that "success breeds success", as success in the next step is proportional to success of the previous step. Formally, such processes are best described by multiplicative stochastic processes (see Bahr and Passerini 1998; Yakovenko and Rosser 2009). They imply very often skewed distribution of the success variable, i.e., a lognormal or a power law distribution (see Prinz et al. 2015). Moreover, what separates "the best" from "the rest" may be that the chain of successful steps is much shorter in case of the latter.

As mentioned by Fox and Kochanowski (2004, p. 518), similar stochastic processes do generally not explain the rationale behind these processes. For the Top 2000 charts, however, there is an economic interpretation of the stochastic process. Leaving aside the economics of the empirical distribution of income and wealth, it will be argued below that there are indeed two processes with respect to the ranking of pop songs. There is a very slow process with very little innovation among the top 100 songs and a much more innovative process at the lower and lowest ranks. Put technically, in the top ranks, the innovation factor $\lambda$ (see Eq. 7 above) is close to zero, whereas it is significantly higher in the lower the ranks. Hence, the top ranked songs are not contestable, in sharp contrast to the remaining songs. With about 50 incontestable songs ( $2.5 \%$ of songs), a very large majority of songs $(97.5 \%$ of songs) compete heavily with each other.

As argued by Prinz et al. (2015), an economic rationale for such a small number of incontestable items as, for instance, artworks and pop songs entails incomplete consumer information about the aesthetic value of cultural goods. As soon as a small number of highly respected institutions are established that define aesthetic value - as in the cases of art galleries and top museums - no further institutions are required. Put differently, a highly variable or very large number of such institutions would cause insecurity to return concerning the aesthetic value of cultural items and hence lead to incomplete information. These institutions define the quality of 
cultural goods. As a consequence, the number of top artists, paintings, etc., will be very limited, because they represent the highest quality standards for the respective goods. Nonetheless, competition between artworks, as well as standard-setting institutions, will remain-but not at the top level. Therefore, a small number of standard-setting institutions, as well as artworks, will be incontestable. The large number of institutions and artworks, however, will continue to compete with each other for art lovers and customers.

However, this explanation of the divided nature of pop song rankings is incomplete. Social institutions emerge because they have a function for society (Parsons 1951; Merton 1957; Luhmann 1995, 2000). The function of pop music is described by Wilfred Dolfsma: "By consuming pop music, people want to express who they are, to which group they belong, what their identity is" (Dolfsma 1999, p. 1019). According to the host radio station, the Top 2000 charts reach about three quarters of the Dutch population each year. It is broadcast between Christmas and New Year's Eve, because at this time of the year, people tend to spend their time in groups (NPO Radio 2 2015a, b). "The chart connects generations but it also unites music genres (...) For that reason, the Top 2000 connects a wide range of music lovers" (NPO Radio 2 2015b).

To fulfill this task successfully, the Top 2000 charts can be considered economically as a coordination game. To be enjoyed by a large part of the Dutch population between Christmas and New Year's Eve, the Top 2000 pop songs have to be truly very popular. To formalize the decision problem of a voter, assume that they gain utility not only form listening to their individually preferred, highly ranked songs, songs $s_{i}$, but also from the number of other people who very much like to listen to these songs, $\# p\left(\right.$ (songs $\left._{i}\right)$ :

$$
u_{i}\left(\operatorname{songs}_{i}, \# p\left(\operatorname{songs}_{i}\right)\right), \quad \frac{\partial u_{i}}{\partial \operatorname{song}_{i}}>0, \quad \frac{\partial u_{i}}{\partial \# p} \frac{\partial \# p}{\partial \operatorname{songs}_{i}}>0 .
$$

The first utility component is individual, and the second is social (see for "social preferences" Bowles and Gintis 2011, chapter 3). Note that the second utility component is defined via the utility that the songs provide others than person $i$, since otherwise, they would not want to listen to these songs. A plausible specification of the utility of songs could be (see, for instance, Dybvig and Spatt 1983, p. 233, in a different context):

$$
u_{i}\left(\operatorname{songs}_{i}, \# p\left(\operatorname{songs}_{i}\right)\right)=\# p\left(\operatorname{songs}_{i}\right) \cdot u_{i}\left(\operatorname{songs}_{i}\right) .
$$

This specification states that the individual utility gained from the songs is proportional to the number of other people who also like them most.

Obviously, choosing songs to maximize utility in Eqs. (8) or (9) requires a guessing of the most preferred songs in a specific country. These songs provide a "focal point" (Schelling 1960, p. 57) as a form of "tacit coordination" (Schelling 1960, p. 54) for the individual voting decision, if it is socially motivated in the sense defined above. ${ }^{7}$

\footnotetext{
7 The neural basis for focal points in coordination games is studied in McMillan et al. (2011).
} 
Since conventions (Young 1996) and culture specifics (Jackson and Xing 2014; Molleman et al. 2013), as well as networks (Tomassini and Pestelacci 2010) and tradition (Bentley and Omerod 2009; Golosovsky and Solomon 2013), play a fundamental role in coordination games, the relevant songs tend to be rather older ones and so-called evergreens, and songs in the country's own language, i.e., in Dutch for the Top 2000. Since the number of these songs is restricted, as well as the number of votes a person has, these songs are likely to collect a huge proportion of all votes. As shown above, the top ranks are almost fixed in advance. This concentration of votes on a small number of extremely well-known songs as a cultural focal point may explain the fat ${ }^{8}$ Pareto or power law tail of the song success distribution.

In contrast, since each voter in the Top 2000 has 10 or 15 votes (depending on the year), there is also leeway for expressing one's own song preferences. Because this voting is unconditional, the whole range of available songs provides the basis for individual selection. The result is far fewer votes for these songs, and a very large spectrum. Hence, individual idiosyncratic preferences may explain the long tail of the song success distribution that follows an exponential distribution. ${ }^{9}$

As a result, "nostalgia," "evergreens" (Sterken), "focal points" (Schelling), "memory" (Dall' Asta L. and Castellano 2007), and "superstardom" (Rosen 1981; Adler 1985; MacDonald 1988) may have the same social function, namely to coordinate the social preferences of people in large coordination games.

Additionally, the host radio station of the Top 2000, NPO Radio 2, took over the role of a coordinator, when it introduced the Top 2000 in 1999. It created a channel for coordinating pop music preferences at a time of the year when people in Western societies are most susceptible to group-feelings. "Whatever memories they are built on: Music carries meaning and songs tell the stories of our lives. The Top 2000 is compiled of thousands of these stories" (NPO Radio 2 2015a, b). By allowing people to choose a large number of songs quite freely, it contributed to a so-called the maximal Nash, i.e., to an equilibrium of the coordination game that dominates all other Nash equilibria for all people (Dybvig and Spatt 1983, p. 234).

\section{Conclusion}

In this paper, rankings are interpreted as results of coordination games. To demonstrate this, the annual Dutch Top 2000 charts are analyzed both statistically and econometrically. The first objective is to find out whether there is a superstar effect and to what extent the song ranking is contestable. To check this, a test is conducted to determine what probability distribution fits best the success of songs in the Top 2000. Knowing the probability distribution, the data-generating stochastic process could be determined. Finally, the parameters of the stochastic process are

\footnotetext{
${ }^{8}$ For the differentiation of "fat" and "thin" tails of distributions, see Sornette (2000, p. 138).

9 See Vilone et al. (2012) for an analysis of the dynamics of social and strategic imitation in an econophysics model. The resulting distribution converges either to an exponential or to a power law tail, depending on the imitation. An evolutionary process of social preferences for music in a sociophysics voter model is developed in Lanoue (2015).
} 
interpreted economically with respect to competition between the songs and the innovation level within the rankings. These results are employed to find out what such rankings are good for. It is argued that these rankings perform a social function of coordinating people in large groups.

The empirical result is that song success in the Top 2000 charts cannot be described by a single empirical distribution, but by two. The top 100-150 ranks follow a Pareto distribution, whereas the songs up to rank 1500 are best fitted by an exponential distribution. Consequently, if one wants to describe the empirical distribution of song success by a single distribution, an exponentially tempered power law (Pareto) distribution seems to be appropriate.

Because of the Pareto distributed song success variable, the underlying stochastic process for the top 100-150 songs is an inhomogeneous geometric Brownian motion. This stochastic process implies almost no change of the ranks and, hence, a very low degree of song contestability. Technically, the innovation factor is close to zero in the top 10 ranks, whereas it is increasing with the position in the rankings. Quantitatively, about 50 songs or $2.5 \%$ are incontestable. This is different for the lower ranks, where song success follows an exponential distribution. For these ranks, songs are much more contestable, and the level of musical innovation is considerable higher. This means that about $97.5 \%$ of the remaining ranks are highly competitive.

Since the ranking in the Top 2000 is based on voting, the question arises as to what kind of voting strategies may generate the respective song rankings. Because of the empirical distribution, a pure random walk can be excluded. Instead, voters are confronted with a typical "beauty contest" (Keynes 1936, p. 156), that is, with a coordination game. Although voters have their own song preferences, in order to get the most enjoyment from listening to the Top 2000 radio broadcasting, they nonetheless want a large number of equally minded listeners. As a consequence, voters have to choose which of these two strategies (or a combination) they would like to apply. The top ranks are then the result of a coordination game in which evergreens, nostalgia, and superstardom play a coordinative role. The remaining ranks, however, can be interpreted as the result of voting according to individual preferences.

As indicated empirically by a study with data of the Eurovision Song Contest, data from this contest can be employed as predictors of trade relations between countries (Kokko and Tingvali 2012). Therefore, different incomes and preferences, as well as cultural affinity, may even be captured by single supranational cultural events. In effect, it demonstrates the close relation between culture and the economy as a whole. Put differently, the economy may be considered as a part of culture.

There is another aspect worth noting. The Top 2000 charts are a nonprofit cultural event, in contrast to other cultural goods as, for instance, books, movies, commercial charts, and sports. The Dutch Top 2000 voting is one of those events that provide evidence for very similar social processes with both for-profit and nonprofit culture goods. As pointed out in The Economist (N. N. 2009), modern technology did not end block busters in the profit-based culture economy, but to the contrary, block busters are currently more important than ever. In addition, however, also the number of culture businesses, as well as competition between and 
innovation within culture businesses, did not decline (N. N. 2009). This development is mirrored in the nonprofit sector of culture, as the Top 2000 charts demonstrate. Voting by approval and by economic demand seems to follow common principles in the case of culture goods.

Last, but not least, the methods applied here to the Top 2000 songs may also be used to analyze other rankings, ranging from scientific journals to universities and scientists. The hypothesis is that all forms of rankings are coordination tools in large-scale coordination games.

Acknowledgments I thank three referees for their comments and suggestions on earlier versions of this paper, as well as Brian Bloch for extensive text editing. However, all remaining errors are mine.

\section{Compliance with ethical standards}

Conflict of interest The author declares that he has no conflict of interest.

Open Access This article is distributed under the terms of the Creative Commons Attribution 4.0 International License (http://creativecommons.org/licenses/by/4.0/), which permits unrestricted use, distribution, and reproduction in any medium, provided you give appropriate credit to the original author(s) and the source, provide a link to the Creative Commons license, and indicate if changes were made.

\section{References}

Adler, M. (1985). Stardom and talent. American Economic Review, 75, 208-212.

Adler, M. (2006). Stardom and talent. In V. Ginsburgh \& D. Thorsby (Eds.), Handbook of the economics of art and culture (pp. 895-906). Amsterdam: North-Holland.

Asai, S. (2011). Demand analysis of hit music in Japan. Journal of Cultural Economics, 35, 101-117.

Bahr, D. B., \& Passerini, E. (1998). Statistical mechanics of opinion formation and collective behavior: Micro-sociology. Journal of Mathematical Sociology, 23(1), 1-27.

Bentley, R. A., \& Omerod, P. (2009). Tradition versus fashion in consumer choice. arXiv:0902.2976v1 [physics.soc-ph].

Bhattacharjee, S., Gopal, R. D., Lertwachara, K., \& Marsden, J. R. (2007). Stochastic dynamics of music album lifecycle: An analysis of the new market landscape. International Journal of HumanComputer Studies, 65(1), 85-93.

Bowles, S., \& Gintis, H. (2011). A cooperative species. Princeton and Oxford: Princeton University Press.

Bradford, S. (1934/1985). Sources of information on specific subjects. Engineering, 137, 85-86 (reprinted in: Journal of Information Science, 10, 176-180).

Bradlow, E. T., \& Fader, P. S. (2001). A Bayesian lifetime model for the "Hot 100" Billboard songs. Journal of the American Statistical Association, 96, 368-381.

Burke, A. E. (2011). The music industry. In R. Towse (Ed.), A handbook of cultural economics (2nd ed., pp. 297-303). Cheltenham; Northampton, MA: Edward Elgar.

Chung, K. H., \& Cox, R. A. K. (1994). A stochastic model of superstardom: An application of the Yule distribution. Review of Economics and Statistics, 76(4), 771-775.

Connolly, M., \& Krueger, A. B. (2006). Rockonomics: The economics of popular music. In V. A. Ginsburgh \& D. Throsby (Eds.), Handbook of the economics of art and culture (Vol. 1, pp. 668-718). Amsterdam: Elsevier.

Cook, K. L. (1989). Laws of scattering applied to popular music. Journal of the American Society for Information Science, 40(2), 277-283.

Cox, R. A. K., Felton, J. M., \& Chung, K. H. (1995). The concentration of commercial success in popular music: An analysis of the distribution of gold records. Journal of Cultural Economics, 19, 333-340.

Crain, W. M., \& Tollison, R. D. (2002). Consumer choice and the popular music industry: A test of the superstar theory. Empirica, 29, 1-9. 
Dall' Asta, L., \& Castellano, C. (2007). Effective surface-tension in the noise-reduced voter model. European Physics Letters, 77, 60005.

DeSerpa, A. C., \& Faith, R. L. (1996). "Bru-u-u-uce": The simple economics of mob goods. Public Choice, 89, 77-91.

Dolfsma, W. (1999). The consumption of music and the Expression of VALUES: A social economic explanation for the advent of pop music. American Journal of Economics and Sociology, 58(4), 1019-1046.

Drăgulescu, A., \& Yakovenko, V. M. (2001). Exponential and power-law probability distributions of wealth and income in the United Kingdom and the United States. Physica A, 299, 213-221.

Dybvig, P. H., \& Spatt, C. S. (1983). Adoption externalities as public goods. Journal of Public Economics, 20, 231-247.

Fenn, D., Suleman, O., Efstathiou, J., \& Johnson, N. F. (2006). How does Europe Make Its Mind Up? Connections, cliques, and compatibility between countries in the Eurovision Song Contest. Physica A, 360, 576-598.

Fox, M. A., \& Kochanowski, P. (2004). Models of superstardom: An application of the Lotka and Yule distributions. Popular Music and Society, 27(4), 507-522.

Gabaix, X., \& Ibragimov, R. (2011). Rank-1/2: A simple way to improve the OLS estimation of tail exponents. Journal of Business \& Economic Statistics, 29(1), 24-39.

García, D., \& Tanase, D. (2013). Measuring cultural dynamics through the Eurovision Song Contest. arXiv:1301.2995v1 [physics.coc-ph].

Gatherer, D. (2006). Comparison of Eurovision Song Contest simulation with actual results reveals shifting patters of collusive voting alliances. Journal of Artificial Societies and Social Simulation, 9(2), 1.

Giles, D. E. (2006). Superstardom in the U.S. popular music industry revisited. Economics Letters, 92, $68-74$.

Ginsburgh, V. (2003). Awards, success and aesthetic quality in the arts. Journal of Economic Perspectives, 17(2), 99-111.

Ginsburgh, V., \& Noury, A. G. (2008). The Eurovision Song Contest. Is voting political or cultural? European Journal of Political Economy, 24, 41-52.

Golosovsky, M., \& Solomon, S. (2013). The transition towards immortality: Non-linear autocatalytic growth of citations to scientific papers. Journal of Statistical Physics, 151, 340-354.

Goris, T. (2010). Wiskunde en de Top 2000. Nieuwe Wiskrant 30-2/December 2010, 39-40.

Haan, M. A., Dijkstra, S. G., \& Dijkstra, P. T. (2005). Expert judgment versus public opinion-Evidence from the Eurovision Song Contest. Journal of Cultural Economics, 29, 59-78.

Hamlen, W. A. (1991). Superstardom in popular music: Empirical evidence. Review of Economics and Statistics, 73(4), 729-733.

Hamlen, W. A. (1994). Variety and superstardom in popular music. Economic Inquiry, 32(3), 395-406.

Hendricks, K., \& Sorensen, A. (2009). Information and the skewness of music sales. Journal of Political Economy, 117(2), 324-369.

Jackson, M. O., \& Xing, Y. (2014). Culture-dependent strategies in coordination games. PNAS, 111(suppl. 3), 10889-10896.

Keynes, J. M. (1936). The general theory of employment, interest, and money. London: Macmillan.

Kokko, A., \& Tingvali, P. G. (2012). The Eurovision Song Contest, preferences and European trade. Ratio Working Paper No. 183, Stockholm.

Lamport, L. (2006). Measuring celebrity. Annals of Improbable Research, 12(1), 14-15.

Lanoue, D. (2015). The iPod model. Electronic Journal of Probability, 20(64), 1-20.

Lotka, A. J. (1926). The frequency distribution of scientific productivity. Journal of the Washington Academy of Sciences, 16, 317-323.

Luhmann, N. (1995). Social systems. Stanford, CA: Stanford University Press.

Luhmann, N. (2000). Art as a social system. Stanford, CA: Stanford University Press.

MacDonald, G. (1988). The economics of rising stars. American Economic Review, 78, 155-166.

Marshall, A. (1947). Principles of economics (8th ed.). London and Basingstoke: Macmillan.

McMillan, C. T., Rascovsky, K., Khella, M. C., Clark, R., \& Grossman, M. (2011). The neural basis for establishing a focal point in pure coordination games. SCAN, 7, 881-887.

Meerschaert, M. M., Roy, P., \& Shao, Q. (2012). Parameter estimation for exponentially tempered power law distributions. Communications in Statistics-Theory and Methods, 41, 1839-1856.

Merton, R. K. (1957). Social theory and social structure. Glencoe, IL: The Free Press. 
Molleman, L., Pen, I., \& Weissing, F. J. (2013). Effects of conformism on the cultural evolution of social behaviour. PLoS One, 8(7), e68153.

N. N. (2009). A world of hits. http://www.economist.com/node/14959982. Accessed February 10, 2016.

Newman, M. E. J. (2010). Networks. An introduction. Oxford: Oxford University Press.

NPO Radio 2 (2014). Top2000. Lijsten alle jaren. http://www.radio2.nl/radio2-2/documents/2014/lijsten_ alle_jaren.xlsx.

NPO Radio 2 (2015a). Top2000 handout. http://www.radio2.nl/ebu/download/top2000-handout-final.pdf. Accessed November 11, 2015.

NPO Radio 2 (2015b). Building blocks. http://www.radio2.nl/ebu/?page=t2k-building-blocks. Accessed November 05, 2015.

Pan, R. K., \& Sinha, S. (2010). The statistical laws of popularity: Universal properties of the box-office dynamics of motion pictures. New Journal of Physics, 12, 115004.

Parsons, T. (1951). The social system. Glencoe, IL: The Free Press.

Prinz, A. (2015). Do capitalistic institutions breed billionaires? Empirical Economics,. doi:10.1007/ s00181-015-1058-0.

Prinz, A., Piening, J., \& Ehrmann, T. (2015). The success of art galleries: A dynamic model with competition and information effects. Journal of Cultural Economics, 39, 153-176.

Rosen, S. (1981). The economics of superstars. American Economic Review, 71, 845-858.

Rosen, S. (1983). The economics of superstars: Reply. American Economic Review, 73, 460-462.

Salganik, M. J., Dodds, P. S., \& Watts, D. J. (2006). Experimental study of inequality and unpredictability in an artificial cultural market. Science, 311, 854-856.

Schelling, T. C. (1960). The strategy of conflict. Cambridge, Massachusetts: Harvard University.

Schulze, G. G. (2011). Superstars. In R. Towse (Ed.), A handbook of cultural economics (2nd ed., pp. 401-407). Cheltenham, UK; Northampton, MA, USA: Edward Elgar.

Simkin, M. V., \& Roychowdhury, V. P. (2013). A mathematical theory of fame. Journal of Statistical Physics, 151, 319-328.

Simon, H. A. (1955). On a class of skew distribution functions. Biometrika, 42, 425-440.

Sornette, D. (2000). Critical phenomena in natural sciences. Berlin: Springer.

Spierdijk, L., \& Vellekoop, M. (2009). The structure of bias in peer voting systems: Lessons from the Eurovision Song Contest. Empirical Economics, 36, 403-425.

Statista. (2015). Statistics and facts about music industry in the U.S. http://www.statista.com/topics/1639/ music/. Accessed November 4, 2015.

Statistics Netherlands. (2015). Population; key figures (as of April 05, 2013). WWW.Statline.cbs.nl.

Sterken, E. (2014). Collective memory and nostalgia in the Dutch Radio2 Top2000 Chart 1999-2013. CESifo Working Paper No. 4632.

Strobl, E. A., \& Tucker, C. (2000). The dynamics of chart success in the U.K. pre-recorded popular music industry. Journal of Cultural Economics, 24, 113-134.

Tomassini, M., \& Pestelacci, E. (2010). Coordination games on dynamical networks. Games, 1, $242-261$.

Vilone, D., Ramasco, J. J., Sánchez, A., \& San Miguel, M. (2012). Social and strategic imitation: The way to consensus. Scientific Reports, 2(686), 1-7.

Yair, G., \& Maman, D. (1996). The persistent structure of hegemony in the European Song Contest. Acta Sociologica, 39, 309-325.

Yakovenko, V. M., \& Rosser, J. B, Jr. (2009). Colloquium: Statistical mechanics of money, income, and wealth. Review of Modern Physics, 81(4), 1703-1725.

Young, H. P. (1996). The economics of convention. Journal of Economic Perspectives, 10(2), 105-122.

Yule, G. U. (1924). A mathematical theory of evolution, based on the conclusions of Dr. J. C. Willis, F.R.S. Philosophical Transactions of the Royal Society, B, 213, 21-87. 